\title{
Genética humana y el valor de los valores no-epistémicos en la restitución de identidad en Argentina
}

\author{
Human Genetics and the Value of Non-epistemic Values \\ for Restituting Identity in Argentine
}

\author{
Livio Mattarollo \\ Universidad Nacional de La Plata \\ Consejo Nacional de Investigaciones Científicas y Técnicas \\ livio.mattarollo@nacio.unlp.edu.ar
}

\begin{abstract}
Resumen
En el marco de la discusión sobre el ideal de ciencia libre de valores, Heather Douglas afirma que en muchos casos los valores tradicionalmente denominados no-epistémicos son necesarios para un buen razonamiento en ciencia. En este artículo propongo retomar su enfoque para examinar la investigación que condujo a la elaboración del "índice de abuelidad", elemento clave en la restitución de identidad de niños/as apropiadas durante la última dictadura en Argentina (1976-1983). Así, los propósitos son (i) reconstruir las principales contribuciones teóricas de Douglas a la discusión, especialmente sus razones para rechazar el ideal y su distinción entre roles directo e indirecto de los valores en ciencia; y (ii) analizar la publicación resultante de la investigación sobre el "índice de abuelidad". La hipótesis que anima el artículo es (iii) que dadas las consecuencias no-epistémicas de la investigación asociadas a su riesgo inductivo, varias de las decisiones metodológicas, en particular respecto a la ponderación de la suficiencia de la evidencia, se explican por referencia a valores sociales, morales y políticos, de modo que dicha investigación es un caso de legítima y necesaria integración entre valores epistémicos y no-epistémicos y que respeta los parámetros de objetividad establecidos por Douglas. Todo ello permite, finalmente, enfatizar la relevancia del aporte de Douglas para abordar las relaciones entre ciencia y política.
\end{abstract}

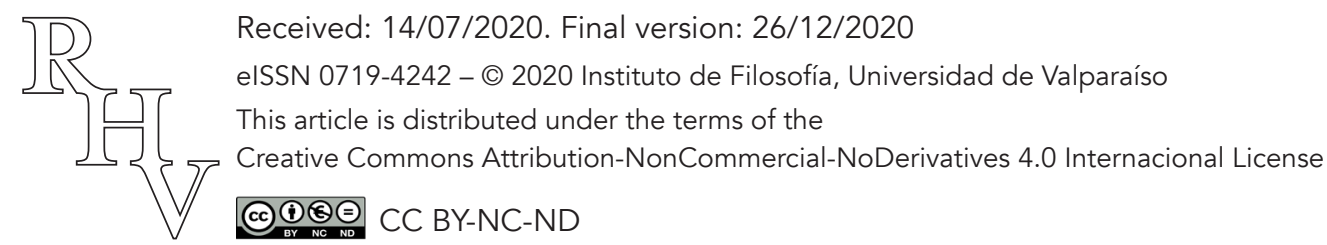


Genética humana y el valor de los valores no-epistémicos en la restitución de identidad en Argentina

Livio Mattarollo

Palabras clave: Heather Douglas, riesgo inductivo, objetividad, índice de abuelidad, derechos humanos.

\begin{abstract}
Within the context of the discussion about value-free science ideal, Heather Douglas claims that in several cases non-epistemic values are needed for good reasoning in science. In this article I aim at recovering her viewpoint in order to examine the research driving to the Genetic Grandparent Inclusion-Probability Index, a crucial element to restitute the identity of children who were abducted during Argentinean dictatorship (1976-1983). Thus, my purposes are (i) to reconstruct Douglas' main theoretical contributions, specifically her reasons to reject the ideal as well as the distinction between direct and indirect roles for values in science; and (ii) to analyze the scientific paper resulting from the "Grandparent-Inclusion Index" research. The main hypothesis is (iii) that several decisions of this research, particularly the establishment of what is to be considered sufficient evidence, should be explained by reference to social, ethical and political values. Both because of the non-epistemic consequences of the inquiry related to its inductive risk and because such inquiry is a case in which there is legitimate and necessary integration between epistemic and non-epistemic values and that it is also a case in which Douglas' criteria of objectivity is reached. By virtue of these reasons, the significance of Douglas' contribution to the analysis of the relationship between science and politics is emphasized.
\end{abstract}

Keywords: Heather Douglas, inductive risk, objectivity, grandparent inclusion, probability index, human rights.

\title{
1. Introducción
}

Cuando la física, la química, la biología, la medicina contribuyen a poner al descubierto los males concretos que sufre la humanidad, y al desarrollo de proyectos encaminados a ponerles remedio, se convierten en ética [...] Pero esa ganancia no queda limitada a la faceta moral. Desde ese momento, las ciencias naturales dejan de estar divorciadas de lo humano, adquieren la cualidad de lo humano.

John Dewey, La Reconstrucción de la Filosofía

En los últimos años la relación entre conocimiento científico y valores tradicionalmente denominados "no-epistémicos" ha sido objeto de un creciente interés filosófico y ha suscitado un arduo debate en torno al ideal de ciencia libre de valores. En principio, y como observa Ricardo J. Gómez $(2014,13)$, en la dimensión "externa" de la ciencia la presencia de valores no-epistémicos parecería obvia; sin embargo, sucedería lo contrario

Revista de Humanidades de Valparaíso, 2020, No 16, 255-275

(c) $(1)(9)$ CC BY-NC-ND 
para la dimensión "interna", de validación del conocimiento -contextos de descubrimiento y justificación, siguiendo la expresión de la filosofía de la ciencia standard. En este marco surgen tres cuestiones fundamentales: (i) ¿la determinación de la validez del conocimiento está efectivamente libre de valores sociales, éticos, políticos? (ii) ¿cómo debería entenderse y articularse la presencia de valores no-epistémicos dada la innegable dimensión pública de la ciencia? y (iii) ¿es legítima y deseable la presencia de valores no-epistémicos en ambas dimensiones de la investigación? Frente a estas preguntas, en términos generales se identifican posiciones que defienden el ideal qua ideal (Lacey 1999); posiciones que lo discuten, sea desde enfoques feministas (Longino 1990; Anderson 1995; Kourany 2010) o desde la identificación del pluralismo teleológico y axiológico de las prácticas científicas (Olivé 2000; Echeverría 2002); y posiciones que además proponen distintas alternativas tales como el ideal de ciencia bien ordenada (Kitcher 2001; 2011) o de social value management (Longino 1990; 2002).

Las interpretaciones sobre la distinción y las relaciones entre valores epistémicos y no-epistémicos suscita perspectivas igualmente diversas: aquellas que mantienen la distinción e incorporan a los valores no-epistémicos como elementos secundarios de la investigación (Steel \& Whyte 2012), aquellas que también mantienen la distinción pero señalan que dados los múltiples objetivos prácticos de la investigación en ciertas ocasiones los valores no-epistémicos deberían tener prioridad (Elliott 2013; Hicks 2014) y aquellas que en última instancia rechazan la distinción (Rooney 1992; Longino 1996). En cuanto partícipe activa de la discusión, la filósofa canadiense Heather Douglas (2000; 2007; 2009; 2014; 2016) avanza por las dos líneas argumentativas típicas del tratamiento del asunto: el argumento del vacio [gap], vinculado con la tesis de la sub-determinación de la teoría por la evidencia disponible, y el argumento del error, relacionado con el riesgo inductivo de toda investigación (sobre los argumentos, Brown 2013). Así, frente a enfoques como el de Lacey -quien interpreta el ideal de ciencia libre de valores en términos de neutralidad, imparcialidad y autonomía y para quien dicho ideal representa una aspiración de las prácticas científicas y un criterio para su evaluación (Lacey 1999, 2, 17-18)-, Douglas considera que el ideal es inalcanzable [unattainable], insostenible [untenable] e incluso un mal ideal que en ciertos casos conduce a una visión inaceptable de la ciencia. Como contrapartida, afirma que en muchos casos los valores no-epistémicos son necesarios para un buen razonamiento en ciencia (Douglas 2007, 121-122).

El objetivo de este artículo es recuperar el marco teórico de Douglas a efectos de analizar la incidencia de valores no-epistémicos en la investigación que condujo a la adaptación del índice de probabilidad de inclusión de parentalidad. Esta adaptación, también llamada índice de "abuelidad", ha resultado un aporte vital para la restitución de identidad de niños/as apropiadas durante la última dictadura cívico-eclesiástico-militar en Argenti- 
na (1976-1983) y ha sido empleada como modelo para trabajos similares en otros países. ${ }^{1}$ En función del objetivo señalado, en primer lugar reconstruiré los argumentos con los que Douglas desafía y a la postre rechaza el ideal de ciencia libre de valores junto con su distinción entre roles directo e indirecto de los valores en ciencia. En segundo lugar, examinaré el artículo "Genética Humana y Derechos Humanos" (di Lonardo et al. 1984), primera publicación científica sobre el referido índice, a fines de identificar qué instancias de la investigación implicaron decisiones metodológicas cruciales para su desarrollo. En tercer lugar, argumentaré en favor de la hipótesis del presente trabajo, a saber: que dadas las consecuencias no-epistémicas de la investigación asociadas a su riesgo inductivo, varias de esas decisiones metodológicas, en particular respecto a la ponderación de la suficiencia de la evidencia obtenida con los distintos marcadores genéticos probados, se explican por referencia a valores sociales, morales y políticos, y que la incidencia de esos valores es indirecta, razón por la que dicha investigación se mantiene dentro de los criterios de objetividad identificados por Douglas. En suma, sostendré que dicha investigación representa un caso de necesaria y legítima integración entre valores epistémicos y no-epistémicos y que su valor es doble pues permite considerar adecuadamente los riesgos de la investigación y revierte favorablemente sobre esta última en tanto funciona como razón para continuar las pruebas hasta obtener el grado de certeza más alto posible. Finalmente, indicaré las razones por las que el enfoque de Douglas permite avanzar en la necesaria reflexión sobre la relación entre ciencia, política y sociedad.

\section{Del desafío al rechazo del ideal}

Siguiendo el análisis de Longino (1990, 83-86) y a tono con lo planteado en la Introducción, Douglas reconoce un sentido de exterioridad de la ciencia para el cual los valores no-epistémicos operan legítimamente al momento de decidir sobre la selección de problemas a resolver, el uso directo del conocimiento científico en la sociedad y las limitaciones en las opciones metodológicas, como por ejemplo testear sobre animales o seres humanos. En efecto, estas etapas involucran valores no-epistémicos porque incluyen consideraciones sobre las consecuencias directas e intencionales de cualquier curso de acción específico. Sin embargo, este modelo no es suficiente para desafiar las posiciones clásicas sobre el papel de los valores en la ciencia pues no considera la incidencia de valores no-epistémicos en el nivel del razonamiento científico (Douglas 2016, 3). Más allá de las reconstrucciones históricas en torno a la conformación del ideal de ciencia

\footnotetext{
${ }^{1}$ En lo que sigue me referiré a las niñas/os y nietas/os como niñas y nietas. Asimismo, tomaré identidad en el sentido de identificación genética de una persona, a sabiendas de que la concepción de identidad personal es multidimensional e incluye diversos aspectos filosóficos, sociales, psicológicos, etc., profundamente trabajados desde cada enfoque.
}

Revista de Humanidades de Valparaíso, 2020, No 16, 255-275 
libre de valores, en lo que sigue me interesa recuperar ciertos argumentos de Douglas en referencia a las condiciones de inclusión de valores no-epistémicos en el referido plano del razonamiento científico. ${ }^{2}$

En principio es importante señalar que Douglas reinterpreta el ideal en términos de valores aceptados y no aceptados en el proceso de inferencia o validación, es decir, como un "ideal de emplear sólo valores epistémicos en la inferencia científica" (Douglas 2016, 3). ${ }^{3}$ Frente al ideal así comprendido, su planteo podría articularse en dos instancias, una de crítica y otra de rechazo. Con respecto a la primera, Douglas elabora tres desafíos distintos pero tendientes a un mismo cuestionamiento general (2016, 3-8). El primer desafío es el descriptivo: existe una dificultad transversal para eliminar los valores del proceso científico y los resultados obtenidos porque sólo se pueden testear aquellas teorías que se tienen a disposición utilizando asunciones contextuales y ambos elementos reflejan los valores de quienes investigan. Este punto se enlaza con el argumento del vacío: si bien la evidencia generalmente no es suficiente para definir qué afirmaciones son adecuadas, para garantizar que las alternativas dispuestas son todas las alternativas plausibles ni para dar cuenta de las asunciones contextuales de la investigación, lo cierto es que efectivamente se hacen afirmaciones con pretensión científica, de modo que el salto entre teoría y evidencia es efectivamente realizado, de alguna u otra forma. El reconocimiento de ese vacío y de que aun así hay afirmaciones científicas permite advertir que aquel es saldado por ciertos valores y que la ciencia, aun si fuera guiada por una "buena" lógica deductiva, de hecho no está libre de valores.

El segundo es el desafío de los límites, aquel que señala la dificultad de hacer una distinción clara entre valores epistémicos y no-epistémicos. En efecto, entre ambos tipos de valores hay cierta porosidad y en incontables ocasiones los valores epistémicos terminan reflejando a los no-epistémicos, de forma que éstos tienen cierta incidencia "codificada" en el ámbito epistémico en tanto sirven como guías o marcos para las elecciones epistémicas. Además, otro de los límites más difíciles de mantener, aún si se quisiera, es el límite entre ciencia y política, especialmente cuando se trata de asesoramiento científico para políticas públicas. En definitiva, si los valores no-epistémicos moldean a los valores epistémicos y si hay una evidente dificultad para separar taxativamente los ámbitos de incumbencia de la ciencia y de la política que impide mantener límites definidos entre valores epistémicos y no-epistémicos, la demarcación estricta entre ambos junto con el planteo acerca de valores aceptables e inaceptables en la validación del conocimiento científico parecen desdibujarse (Douglas 2004a).

\footnotetext{
${ }^{2}$ Para un análisis histórico de la consolidación del ideal de ciencia libre de valores contemporáneo y del perfil de científico apolítico funcional a las pretensiones militares y expansivas estadounidenses en el marco de la Guerra Fría, ver Reisch (2009) y Douglas (2009).

${ }^{3}$ Las citas de Douglas tienen traducción propia.
} 
El tercer desafío es el normativo, referido al ideal en cuanto ideal. Douglas identifica tres bases distintas para sostener su crítica. Por un lado, la sub-determinación de la teoría por la evidencia y el riesgo inductivo endémico a la práctica científica. Por otro lado, la responsabilidad de las científicas, habida cuenta de que no hacen ciencia para ellas solas sino para la sociedad en la que se inscriben (y de la que, en muchos casos, reciben fondos públicos) y de que tienen autoridad epistémica prima facie sobre sus áreas de investigación, de modo que adquieren un tipo particular de responsabilidad al momento de ponderar las consecuencias de sus errores -de los que nunca estarán absolutamente exentos, dado el riesgo inductivo endémico. Finalmente, la poca adaptabilidad del ideal de ciencia libre de valores a situaciones distintas. En efecto, si en ciertas oportunidades el ideal es demasiado restrictivo al excluir consideraciones valorativas, en otras oportunidades es demasiado flexible pues habilita que ciertos valores epistémicos adquieran un rol preeminente y directo en distintas instancias en las que no deberían hacerlo, por ejemplo durante la interpretación de evidencia: "no queremos -señala Douglas- que los científicos rechacen teorías sólo porque no son simples, o no tan amplias como sus competidoras, o porque parecen tener menos capacidad explicativa." $(2009,103)$.

Como puede advertirse, el desafío de los límites y el desafío normativo abren paso al argumento del error y a la pregunta por cuánta incertidumbre es aceptable en la práctica científica. Recordemos brevemente que el apoyo teórico del argumento del error es la noción de riesgo inductivo planteada por Rudner (1953) y Hempel (1965). En pocas palabras, el riesgo inductivo es el riesgo de error al aceptar o rechazar una hipótesis. En efecto, cuando se aplican criterios de aceptación de hipótesis específicas surgen dos situaciones problemáticas distintas: (i) aceptar una hipótesis falsa y (ii) rechazar una hipótesis verdadera. Luego, es absolutamente importante formular reglas o criterios adecuados para sopesar las consecuencias de los errores al aceptar o rechazar la hipótesis. Según Douglas, es posible implementar esta noción de riesgo inductivo en las tres etapas principales del procedimiento científico: elección de la metodología, recopilación y caracterización de la evidencia e interpretación de los datos. En la medida en que se podrían tomar decisiones equivocadas y propiciar consecuencias indeseables en cada nivel, el concepto de riesgo inductivo podría considerarse en referencia a todas las etapas mencionadas anteriormente. Una decisión es ciertamente apropiada sólo después de esa evaluación sobre las posibilidades y riesgos del error, de modo que, si bien no son determinantes, los valores adquieren un rol de suma importancia en las decisiones científicas relativas a las instancias previamente señaladas (Douglas 2007, 123).

En este marco me interesa subrayar que para Douglas " [...] expandiendo lo que consideramos como riesgo inductivo relevante, el rol potencial de los valores no epistémicos también se expande" $(2000,565)$. El punto crucial es que cuando la ponderación del riesgo inductivo requiere la consideración de las consecuencias no epistémicas de la investigación, dicha ponderación incluye legítimamente valores no-epistémicos en cada uno de los niveles internos de la ciencia en función de determinar los riesgos que se asumen 
Genética humana y el valor de los valores no-epistémicos en la restitución de identidad en Argentina

Livio Mattarollo

o las decisiones que se toman. Luego, la inclusión de los valores no-epistémicos debe ser parte de la definición normativa de ciencia y el rechazo al ideal de ciencia libre de valores deviene explícito:

[...] los valores no epistémicos son una parte necesaria de los aspectos internos del razonamiento científico para casos en los que el riesgo inductivo incluye el riesgo de consecuencias no epistémicas. En estos casos, la ciencia libre de valores es una ciencia inadecuada; el razonamiento es defectuoso e incompleto. Por lo tanto, el standard normativo debe ser reconsiderado. Para la ciencia que tiene claros impactos no epistémicos, ser 'libre de valores' no es un objetivo loable. (Douglas 2000, 559-560).

Todas estas observaciones dan cuenta del "terreno moral de la ciencia" (Douglas 2014). En efecto, una de las consecuencias de haber rechazado el ideal de ciencia libre de valores es la necesidad de reconsiderar las obligaciones morales de quienes investigan. En este contexto, la pregunta crucial es si las responsabilidades específicas del rol científico tienen algún tipo de prioridad por sobre las responsabilidades generales o si por algún motivo quienes llevan adelante las investigaciones deberían quedar exentos de las responsabilidades generales. Douglas (2009, 71-79) articula dos argumentos. En primer lugar, hay muchos casos en los que la pretendida neutralidad moral ha sido o hubiera sido dañina para la sociedad en su conjunto (basta mencionar el Proyecto Manhattan para la bomba atómica). En segundo lugar, no parece haber argumentos convincentes para que quienes investigan tengan cierta excepción moral acerca de la consideración de las consecuencias de su trabajo porque las ponderaciones de las consecuencias de los errores no se realizan al final sino durante la investigación, a medida que sus avances abren distintos escenarios con sus respectivos márgenes de tolerancia al riesgo de error, y son esas mismas personas quienes están mejor capacitadas para evaluar esas consecuencias. En suma, si la responsabilidad moral general se vincula con la ponderación del riesgo de error de las elecciones deliberadas, si el riesgo de error es endémico a la práctica científica y si a niveles de complejidad altos el riesgo sólo puede ser identificado por los propios científicos, entonces tanto las responsabilidades generales como las específicas son necesarias para llevar adelante la investigación de forma correcta (Douglas 2014, 971). Finalmente, es importante señalar que las bases de la responsabilidad de quienes hacen ciencia surgen de reconocer el valor de la ciencia para la sociedad pero al mismo tiempo de reconocer que la sociedad presenta otras fuentes de valor que siempre se deben tener en cuenta. Esto incluye, por ejemplo, la posibilidad de restringir cierto tipo de investigaciones si se oponen a los valores políticos o morales de la sociedad en la que se desarrolla o si pueden sentar bases para posteriores desarrollos dañinos, siempre bajo condición de no dañar el valor general de la ciencia en la sociedad.

Revista de Humanidades de Valparaíso, 2020, No 16, 255-275

(c) $(1)(9)$ CC BY-NC-ND 
Genética humana y el valor de los valores no-epistémicos en la restitución de identidad en Argentina

Livio Mattarollo

\section{La estructura de los valores en ciencia: roles directo e indirecto}

Según ha sido indicado, Douglas reinterpreta el ideal de ciencia libre de valores en términos de valores aceptados o no aceptados en el contexto de validación de las teorías. Ahora bien, su rechazo al ideal torna necesaria la elaboración de un nuevo criterio de análisis. Aquí es donde Douglas introduce una de las novedades más interesantes en la discusión pues propone dejar de referir al tipo de valores aceptados en la práctica científica y distinguir el rol que juegan en las distintas instancias de la investigación, sea un rol directo o indirecto. Así, en su rol directo, los valores actúan como razones que determinan por sí mismos las decisiones en el curso de la investigación. En su rol indirecto, los valores actúan para sopesar la importancia de la incertidumbre con relación a la afirmación que se analiza o a la decisión que se toma, pero no determinan la decisión por sí solos. Lo particularmente interesante es que los tres tipos de valores cognitivos, morales y sociales pueden tener incidencia directa o indirecta en todas las instancias de la investigación, de modo que el desafío central es mantener cierto tipo de límites acerca del rol directo de los valores en ciencia. Si bien el énfasis queda efectivamente puesto en la diferenciación de roles, Douglas también hace ciertas observaciones respecto del tipo de valores cuando señala que prefiere dejar de lado la categoría de valores epistémicos en favor de valores cognitivos. En efecto, los valores epistémicos no son valores propiamente dichos sino más bien criterios básicos que cualquier investigador debe cumplir, de modo que no intervienen al momento de decidir; los valores cognitivos, en cambio, ayudan o asisten al proceso de cognición en ciencia -y es precisamente esa función de guía lo que permite considerarlos como valores. Por su parte, los valores morales o éticos refieren a lo bueno o correcto al momento de considerar las consecuencias del error, mientras que los valores sociales -y me permito agregar, políticos- se vinculan con lo que cierta sociedad valora de hecho: la justicia, la privacidad, la libertad, la innovación, etc. (Douglas 2009, 92-95).

En línea con lo señalado anteriormente, las etapas iniciales de la investigación admiten una incidencia directa de valores. En tales casos, más allá de los conflictos que puede haber entre los tres tipos de valores, la decisión es finalmente tomada a partir de las razones ofrecidas por esos valores, es decir a partir de su incidencia directa. Por supuesto, esto no implica que toda incidencia directa en dichas etapas iniciales sea aceptable. Por ejemplo, seleccionar un problema y una metodología determinados cuya combinación predetermine o restringa el resultado de la propia investigación dañaría la confiabilidad de la ciencia. En suma, resulta claro que algún tipo de rol directo es aceptable, que todo el espectro de valores es relevante y que permitir su incidencia directa no debe socavar el valor de la propia ciencia en cuanto producción de conocimiento confiable. Esto último se evidencia aún más si se considera el rol directo en el núcleo de la actividad, es decir, la caracterización de los datos, la interpretación de la evidencia y la aceptación o rechazo de teorías. En estas instancias Douglas es taxativa: "Si nos importa el conocimiento confiable, entonces los valores no pueden tener un rol directo en aquellas decisiones que surgen una vez que el estudio está en desarrollo." $(2009,102)$.

Revista de Humanidades de Valparaíso, 2020, No 16, 255-275

(c) $(1)(9)$ CC BY-NC-ND 
El rol indirecto de los valores busca morigerar esta incidencia directa en el desarrollo de la investigación, incidencia que según Douglas minaría el valor mismo de la práctica científica. La apelación a valores cognitivos, morales y sociales se vincula con el análisis respecto de la suficiencia de la evidencia, de la evaluación de la incertidumbre y de las consecuencias del error, pero siempre circunscripto a su rol indirecto, particularmente en lo que refiere a las afirmaciones en base a la evidencia disponible. Bajo todas estas consideraciones, elaborar un nivel de significancia estadística permitirá establecer el balance entre falsos positivos y negativos en función de cómo se valoren cada una de estas alternativas. Finalmente, y atendiendo a que la pregunta por qué consecuencias o errores es preferible evitar implica consideraciones no-epistémicas, pues muchas de sus consecuencias son relevantes en otros ámbitos más allá de la propia investigación, queda claro que sopesar todas las posibles consecuencias de error y cursos de acción alternativos supone incluir valores no-epistémicos. Por tanto, en la medida en que la caracterización e interpretación de la evidencia y la decisión respecto de la suficiencia de la evidencia para sostener una hipótesis incluye la incidencia indirecta de valores sociales o morales, la instancia de elección entre hipótesis o teorías alternativas también la incluirá. Sin embargo, aun cuando la ponderación valorativa sea necesaria, en todos estos casos la incidencia de valores debe seguir siendo indirecta pues tienen un rol que cumplir sólo debido a la incertidumbre endémica del conocimiento científico. Como indica Brown $(2013,834)$, el argumento del vacío y el argumento del error suponen que los valores epistémicos tienen prioridad léxica y que los valores no-epistémicos entran en juego sólo cuando la base empírica no es suficiente para sostener la hipótesis o sopesar la evidencia. En este marco resultaría crucial mantener el rol indirecto de los valores no-epistémicos pues caso contrario se estaría minando la rigurosidad de la ciencia. En palabras de Douglas, "[t]oda ciencia está cargada de valores. Pero la ciencia politizada, inaceptable, ocurre cuando se permite que los valores dirijan las afirmaciones empíricas hechas por los científicos." $(2009,113)$.

Por supuesto, la distinción entre roles directo e indirecto de los valores en ciencia no ha quedado exenta de cuestionamientos. Elliott (2011) señala que no resulta claro si la distinción refiere a un orden lógico, a una distinción entre actitudes epistémicas o al tipo de consecuencias que es importante considerar (sean intencionales o no). Además, plantea que la distinción tiene una efectividad limitada pues la interpretación lógica y la consecuencial comparten una debilidad: pueden llegar impedir que los investigadores consideren consecuencias que aceptablemente deberían considerar (Elliott 2013). Por su parte, Steel \& Whyte (2012) indican que la distinción de Douglas no permite advertir incidencias apropiadas e inapropiadas de valores no-epistémicos y proponen un ideal de valores-en-ciencia tal que la incidencia de valores no-epistémicos no entre en conflicto con valores epistémicos.

En mi interpretación, la posición de Douglas presenta otros dos puntos que ameritan consideración. Por un lado, como bien señala Anderson (2004), la discusión sobre el 
ideal de ciencia libre de valores es deudora de la concepción misma de valor y de cómo se interpreta el vínculo entre juicios científicos y juicios valorativos. Ahora bien, Douglas no define con claridad qué entiende por valores. En una de sus pocas indicaciones al respecto, leemos que "[1] os valores son declaraciones de normas, objetivos, deseos [...] La prohibición de Hume se mantiene en su efecto: no se puede derivar un debe de un es." $(2007,126)$. A partir de esta definición podría señalarse que el referido espectro de "normas, objetivos, deseos" es demasiado amplio, de forma que si se pone el énfasis en los valores como declaraciones de deseos sin cualificación ulterior parecería arribarse a una posición subjetivista, mientras que si se pone el énfasis en los valores como declaraciones de normas aquellos parecerían ser una mera repetición de lo dado, sea a nivel institucional o habitual. En cualquier caso, no parece haber una elaboración detallada del concepto de valor-elaboración central al momento de considerar el vínculo entre ciencia y valores. Además, el argumento parece suponer un "fondo" de hechos que idealmente sería suficiente para determinar todas las cuestiones que fueran necesarias en la dimensión interna de la investigación y que debería ser preservado de la incidencia directa de aspectos subjetivos como parecen ser los valores, so pena de dar lugar a una ciencia politizada (en el sentido negativo del término empleado por la autora). ${ }^{4}$

Esta observación abre las puertas al segundo punto a considerar: los valores no-epistémicos quedan siempre relegados a un rol secundario. Dicho de otro modo, parecería que la incorporación de valores no-epistémicos en la dimensión interna de la investigación es sólo por defecto. Cabría preguntarse entonces si Douglas aceptaría la incidencia de valores éticos y sociales en el hipotético caso de absoluta certeza. Según entiendo, Douglas queda comprometida con la primera opción precisamente porque queda apegada a una noción poco detallada de valor -situación que no sería un problema si Douglas estuviera trabajando "solamente" a nivel descriptivo pero que se convierte en una dificultad al momento de elaborar un ideal normativo respecto de la incidencia de los valores en ciencia. No obstante, considero que su enfoque captura un aspecto de vital importancia: no toda incidencia de valores no-epistémicos es deseable para las prácticas científicas, dada la amenaza siempre latente del wishful thinking, pero en algunos casos dicha incidencia se torna deseable e incluso indispensable para el buen razonamiento en ciencia.

\section{4. "Genética humana y derecho humanos": una interpretación filosófica}

Antes de comenzar con el análisis propuesto es indispensable señalar el contexto histórico en el que se desarrolla la investigación que propongo examinar. Entre 1976 y 1983, Argentina sufrió una dictadura cívico-eclesiástico-militar involucrada en crímenes de

\footnotetext{
${ }^{4}$ En otra ocasión -y en línea con Brown $(2013,836)$ - he argumentado que el enfoque cognitivista-experiencial de John Dewey respecto del asunto de los valores puede ofrecer un valioso aporte al argumento de Douglas (Mattarollo 2020).
}

Revista de Humanidades de Valparaíso, 2020, No 16, 255-275 
Genética humana y el valor de los valores no-epistémicos en la restitución de identidad en Argentina

Livio Mattarollo

lesa humanidad y en gravísimas violaciones a los derechos humanos. En el marco de un exterminio sistemático, el gobierno de facto ha sido responsable de la desaparición forzada de 30.000 disidentes políticos, activistas, estudiantes, etc., torturados en centros de detención clandestinos, asesinados e incinerados, arrojados al mar o enterrados como $\mathrm{NN}$ en fosas comunes. Las mujeres embarazadas secuestradas fueron obligadas a entregar a sus bebés en condiciones humillantes y en la mayoría de los casos fueron asesinadas inmediatamente después, lo que resultó en la apropiación de alrededor de 500 niñas y la subsiguiente supresión de su identidad. Como señala Penchaszadeh $(2015,208)$, la apropiación de hijas de personas secuestradas y torturadas fue parte del plan de genocidio y estuvo apoyada en las ideas del psiquiatra español fascista Juan Antonio Vallejo Nájera, para quien las ideas comunistas afectarían el biotipo y por eso esas niñas deberían ser apropiadas y entregadas a familias de "buena moral".

Durante esos años, los familiares de las víctimas se nuclearon en organizaciones de derechos humanos como Madres de Plaza de Mayo y Abuelas de Plaza de Mayo, reconocidas internacionalmente por su inclaudicable tarea de denuncia y búsqueda de la verdad. Con la recuperación de la democracia se creó la Comisión Nacional de Personas Desaparecidas y se elaboró la primera investigación de los crímenes perpetrados durante la dictadura (CONADEP 1984). El gobierno de Raúl Alfonsín solicitó la colaboración de la American Association for the Advancement of Science para ayudar a identificar restos humanos y a restituir la identidad de niñas apropiadas. Después de varios meses de investigación, en diciembre de 1984 Ana di Lonardo, Pierre Darlu, Max Baur, Cristian Orrego y Mary-Claire King publicaron "Genética humana y derechos humanos", un artículo determinante que anunciaba el cálculo de un índice de probabilidad de inclusión de parentalidad o «índice de abuelidad» (di Lonardo et al. 1984). ${ }^{5}$

Teniendo en cuenta que los padres de las niñas se presumen muertos o están desaparecidos, el objetivo de la investigación fue adaptar el índice de paternidad para comprobar si una persona está genéticamente relacionada con un grupo dado de posibles abuelos. Tal y como se indica en la sección sobre la metodología (di Lonardo et al. 1984, 340-344), en primer lugar la investigación se centra en una familia hipotética de una niña apropiada, cuyo conjunto de abuelas y abuelos viven y cuya madre y padre están desaparecidos y presumiblemente muertos. En este punto, se explica que se ha trabajado con marcadores genéticos cuyos alelos presentan un grado especialmente alto de variabilidad porque si solo se toman en cuenta la combinación de alelos presentes en los abuelos y el hipotético nieto, existe la posibilidad de que compartan las formas del gen simplemente por casualidad, y esto es, por supuesto, una posibilidad que la responsabilidad de un buen razonamiento empírico no permite (di Lonardo et al. 1984, 341). Bajo esta premisa y dado que al momento no se contaba con la tecnología necesaria para estudiar secuencias de ADN aunque sí algunos productos génicos, se abría la posibilidad de realizar diferentes

${ }^{5}$ Las citas a di Lonardo et al. (1984) tienen traducción propia.

Revista de Humanidades de Valparaíso, 2020, No 16, 255-275

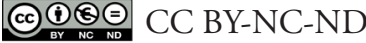


pruebas con marcadores independientes: grupo sanguíneo (factor Rhesus/RH y MN) y antígenos de leucocitos humanos (HLA-A, B, DR). Como lo demuestran las fórmulas, si solo estuviera disponible el análisis basado en el factor $\mathrm{RH}$, para el caso de las primeras nietas hipotéticas, el índice habría sido del 53\%, dado que los haplotipos compartidos son comunes en la población. Del mismo modo, si se hubiera utilizado sólo MN, el índice habría sido del 58\% (di Lonardo et al. 1984, 344-345). Estos índices no son suficientes para afirmar que una persona determinada es nieta de la familia, por lo que hubo necesidad de incorporar marcadores genéticos distintos, teniendo en cuenta que una persona hereda una copia de cada gen de sus abuelos maternos y otra de sus abuelos paternos.

Considerando sólo un gen en la familia presentada anteriormente, después de los cálculos requeridos para el primer marcador seleccionado el índice de probabilidad de que la persona sea nieta de estos abuelos es del $66.7 \%$. Después de una segunda prueba con un marcador genético independiente en la misma familia y la combinación de los dos resultados, el índice de abuelidad es del 96.2\% (di Lonardo et al. 1984, 342). El siguiente paso fue la introducción de marcadores vinculados, como los antígenos de histocompatibilidad HLA. Estos marcadores son los más informativos porque "consisten en [...] dos o más genes estrechamente vinculados en el mismo cromosoma, por lo que se heredan como una unidad", de modo que sus combinaciones posibles en un individuo son tantas que es casi imposible que dos personas no emparentadas presenten la misma combinación (di Lonardo et al. 1984, 342). La incorporación de dichos marcadores vinculados también es crucial para los casos en que uno o más abuelas/os de las nietas hipotéticas están muertas/ os. En estos casos, la técnica depende de la detección de un alelo raro compartido por la niña y la abuela viva. Para las pruebas realizadas con el marcador HLA, es posible definir si una persona es nieta de una familia con un $99.9 \%$ de probabilidad. Por lo tanto, "[c] laramente, sólo HLA es adecuado para confirmar la niñez en esta familia. Los otros marcadores proporcionan poca información adicional" (di Lonardo et al. 1984, 344). Bajo esta hipótesis, en 1984 fue posible confirmar que Paula Eva Logares es nieta de la familia Logares-Grinspon. El caso de Paula es la primera identificación y restitución de una niña secuestrada mediante el uso de esta nueva técnica (aun cuando ella no fue la primera nieta restituida). Ya en 1987 se creó el Banco Nacional de Datos Genéticos, cuya función estuvo desde siempre ligada a los casos de restitución de identidad aplicando técnicas de genética forense. Sobre la base de la técnica desarrollada por di Lonardo et. al. y el posterior desarrollo tecnológico que ha permitido sumar el análisis de ADN mitocondrial, Abuelas de Plaza de Mayo lleva un registro de 130 nietas restituidas, una gesta de altísimo valor científico y político (Abuelas de Plaza de Mayo 2020).

Ahora bien, la forma en que he presentado la investigación no es precisamente el orden en que fue publicada en el artículo científico. Para reconstruir la investigación he consultado entrevistas a Víctor Penchaszadeh, genetista argentino obligado a abandonar el país durante la dictadura y contribuyente crucial a la formulación del índice de abuelidad (cf. Wullf 2008, 44 y ss.). El punto central es que si se consideran los índices obtenidos 
de las diferentes pruebas, señala Penchaszadeh, incluso el $96.2 \%$ de probabilidad no era suficiente para afirmar que una persona es nieta de una familia en particular. Este es un número extremadamente alto que se aceptaría en casi todas las investigaciones científicas como un índice de prueba, pero no en esta en particular. ¿Cómo, entonces, es posible explicar esto?

Como plantea Douglas, la decisión respecto de qué niveles de error se tolerará o qué tipo de errores se permitirán en vistas a la subsiguiente elaboración de un criterio de suficiencia de evidencia basado en cómo se valoren los efectos de las consecuencias y cuál se considere más importante de evitar constituye un ejemplo claro de las ponderaciones surgidas por referencia al riesgo inductivo de toda investigación (Douglas 2000, 565569). A la luz de este enfoque, resulta evidente que di Lonardo et al. están tratando de evitar tanto como sea posible los casos de falsos positivos, es decir, evitar afirmar que una persona es nieta de una familia cuando en realidad no lo es. En efecto, dados los antecedentes históricos y el tipo de consecuencias no-epistémicas derivadas de la investigación, el riesgo de un falso positivo es extremadamente alto. Es por ello que el riesgo no se pondera solamente sobre la base de valores epistémicos sino que incluye consideraciones de orden moral, social y político. Este tipo de casos ilustra las dificultades para eximir a los científicos de las consideraciones valorativas en las afirmaciones empíricas porque (i) la aplicación de sus investigaciones requiere de sus interpretaciones, siempre atravesadas por valores y asunciones contextuales; (ii) la ponderación de las consecuencias no se realiza una vez finalizada la investigación sino que son parte integral de todo el proceso pues en todo el proceso se toman decisiones; y especialmente (iii) delegar las valoraciones significaría atentar seriamente contra la autonomía de los científicos y contra la actividad reflexiva respecto de las consecuencias de la investigación. Además, también ilustra las dificultades para distinguir cabalmente entre "evaluación del riesgo" como prerrogativa exclusivamente científica y valorativamente neutral y "manejo del riesgo" como cuestión exclusivamente política -argumento propio de quienes aún replican que los científicos no tienen que involucrarse en juicios de valor no epistémicos y que se los debe dispensar de la obligación de considerar el manejo de las consecuencias, dejando ese análisis exclusivamente en manos de la política.

En primer lugar, los delitos de desaparición forzada y supresión de identidad son delitos de lesa humanidad y las convenciones internacionales de derechos humanos establecen la obligación del Estado de investigarlos (Naciones Unidas 1993). En ese sentido, tanto por su obligación como por su compromiso, el Estado argentino es responsable de todas las decisiones sobre el proceso de restitución, desde tomar el pedido de las muestras hasta la prueba y decidir la custodia de los nietos recuperados, y también de todas las políticas públicas relativas al tema. A partir de 1984, el Estado argentino implementó políticas públicas para la restitución de identidad y el juicio de los militares, entre ellas la promoción de investigaciones como las de di Lonardo et. Al (1984). Después de varios 
años de interrupción de las investigaciones, entre 2008 y 2015 "Memoria, Verdad y Justicia" fueron los tres valores principales que guiaron las políticas estatales argentinas con respecto al tema.

En segundo lugar, con relación a algunas cuestiones morales, es absolutamente importante considerar no solo el derecho sino también el valor de la identidad. Las nietas deben saber quiénes son, quiénes son sus padres, qué sucedió con ellos y con su familia. Conocer la verdad de su historia de vida es un aspecto crucial para definir su identidad. Hay varios casos en que las niñas han sido adoptadas de buena fe y después de la restitución continúan relacionados con sus familias adoptivas. Sin embargo, en la mayoría de los casos, las niñas apropiadas reaccionan severamente contra quienes las secuestraron. En cualquier situación, llegar a esa verdad "genética" es esencial para todo el proceso de restitución de identidad y, como señala Penchaszadeh, "para aquellos que ya sabían que no eran hijos biológicos de quienes los estaban criando, enterarse que sus padres no los habían abandonado y que su familia los había buscado incansablemente, fue un factor clave en el proceso de curación." $(2015,209){ }^{6}$

En tercer lugar, tanto los aspectos políticos como los éticos -y también de técnica jurídica, que quedan fuera del alcance de estas reflexiones- están estrechamente relacionados con un profundo sentido de la justicia. Esto incluye juzgar y sentenciar a los militares, informar a las familias de los desaparecidos y darles a las niñas apropiadas la oportunidad de conocer a sus parientes biológicos para reconstruir su identidad y las relaciones con su familia biológica. Además, buscar la verdad sobre las niñas secuestradas es de suma importancia en el proceso de construir una memoria histórica, tanto para reparar los horrores sociales de la dictadura como para ser siempre conscientes del valor del estado de derecho. El lazo indispensable entre las investigaciones científicas y las políticas públicas llevadas a cabo permiten considerar, siguiendo a Douglas, que los límites entre valores epistémicos y no-epistémicos resultan porosos y que, por tanto, no es posible establecer una dicotomía entre ellos. En este caso específico, el vínculo filosóficamente deseable entre ciencia y política es patente. Como señala Douglas, la ciencia empleada en la formulación de políticas públicas no debería ser libre de valores pues “[c]ualquier límite, ontológico, procesal u otro, que intente dividir la ciencia y la política sobre la base de la presencia de valores en la política y la ausencia de valores en la ciencia está condenado al fracaso." (2004a, 240).

Ahora bien, para definir la legitimidad de la incidencia de estos valores no-epistémicos resulta crucial elucidar el rol que han tenido. Nuevamente, en las instancias iniciales de la investigación la incidencia de estos valores ha sido directa. De hecho, la investigación se inicia por decisión del Estado argentino y por la invaluable tenacidad de Abuelas de Plaza

\footnotetext{
${ }^{6}$ Como ha sido señalado, la identidad personal no se agota en la identificación genética. Respecto de la construcción parental, política, legal y social de la identidad de nietas apropiadas, ver Amantze Regueiro (2010).
} 
de Mayo. El punto crítico surge entonces al momento de analizar la razón del rechazo del trabajo con marcadores genéticos que ofrecen una probabilidad de confirmación de la hipótesis de un 96,2\% y la exhortación a continuar con la investigación hasta obtener una combinación de marcadores genéticos que arroje un 99,9\% de probabilidad de confirmación de la hipótesis. En mi interpretación, haber descartado un marcado que arroja un índice de confirmación mayor al $96 \%$ obedece a la centralidad de los valores políticos, sociales y morales mencionados en la valoración de las consecuencias no-epistémicas de un falso positivo. Sin embargo, la incidencia de dichos valores no-epistémicos no es directa, es decir que no funcionan como razones en sí mismas para determinar el resultado de las experimentaciones, sino que establecen el marco para considerar la tolerancia al error. En virtud de lo dicho, la incidencia de estos valores no-epistémicos es legítima porque respeta los parámetros establecidos por Douglas y es necesaria porque sin ella no habría una adecuada ponderación de las consecuencias de la investigación. Finalmente, el valor de dicha incidencia radica no sólo en inscribir a la investigación en un marco ético-político más amplio articulado precisamente sobre valores de ese tenor-más allá de las tensiones propias del período de reconstitución de la democracia en Argentina- sino que revierte favorablemente sobre la investigación porque motorizó su continuidad hasta obtener el mayor grado de certidumbre epistémica posible. Esta conclusión tal vez resulte aún más clara si se consideran los pocos casos de falso positivo, entre ellos el de Juliana Inés Trevino y la familia Fontana-Sandoval. Juliana había sido entregada anónimamente a Casa Cuna Buenos Aires y adoptada por una pareja sin relaciones con oficiales militares. De hecho, la propia pareja se acerca a Abuelas de Plaza de Mayo con la sospecha de que Juliana fuera hija de desaparecidos. Tras un primer test con probabilidad de inclusión de $99,81 \%$ sumado a información circunstancial, en 1988 y por orden judicial Juliana fue ubicada con la familia Fontana-Sandoval. No obstante, y debido a la insistencia por parte de la familia Trevino, otro juez ordenó nuevos estudios basados en el análisis directo del material genético que determinaron que Juliana no es nieta de la familia Fontana-Sandoval -razón por la que, por supuesto, Juliana volvió con su familia adoptiva. Más allá de las explicaciones técnicas (cf. Penchaszadeh 1992, 300), el punto que me interesa subrayar es que el análisis de la suficiencia de la evidencia no se realiza exclusivamente con valores epistémicos sino que legítimamente incluye valores no-epistémicos en su rol indirecto, aquellos involucrados en la ponderación moral, política, legal, etc. de las desafortunadas y extremadamente complejas consecuencias no-epistémicas de este tipo de casos.

Más allá de que esta incidencia fuera indirecta, resta hacer frente a la reiterada objeción de que incluir valores no-epistémicos atentaría contra la objetividad de la investigación, suponiendo que se pudiera equiparar objetividad con libertad valorativa en ciencia. Frente a esta estrategia, Douglas plantea que no hay relación necesaria entre objetividad y libertad valorativa. Para ello reformula el concepto de objetividad de forma que mantenga un rol central a la hora de analizar las prácticas científicas, que no pierda densidad teórica y que observe las conclusiones obtenidas respecto de los roles directo e indirecto de los 
valores cognitivos, morales y sociales en la investigación. Así, identifica distintos sentidos de objetividad no reductibles entre sí ni a un sentido único (Douglas 2004b; 2007). En este contexto, y apoyada en algunas consideraciones de Daston (1992), Douglas se concentra en aquellos sentidos cuyas definiciones permiten definir operativamente si la existencia de una entidad o la conclusión de una investigación son objetivas, es decir, en las afirmaciones cognitivas, de modo que su preocupación no está motivada por algunos problemas gnoseológicos clásicos como el problema del escepticismo, del relativismo o del cientificismo, sino que apunta a obtener un sentido "operacionalmente útil" de objetividad que oriente en la correcta toma de decisiones durante la investigación y explicite la fuerza normativa del término (Douglas 2004b, 454).

Así, es posible distinguir ciertos sentidos vinculados con la metodología empleada para dar cuenta de la validez del conocimiento, otros sentidos vinculados con la "postura" de quienes investigan y finalmente otros sentidos vinculados con la evaluación de la investigación por pares. En principio, resulta evidente que la investigación sobre el índice de abuelidad cumple las condiciones de objetividad manipulable y convergente, aquellas que remite a la suficiente aprehensión [sufficiently gotten] de un objeto para emplearlo como instrumento e intervenir exitosamente en el mundo y a la aproximación a un objeto distintos métodos independientes a fines de asegurar la objetividad respecto de las creencias acerca de su existencia o de la confiabilidad de las conclusiones de la investigación. Por otro lado, también resulta evidente que la investigación cumple las condiciones de objetividad relativas a la evaluación por pares, aquella que garantiza el cumplimiento de los criterios científico-académicos. En este marco, Douglas diferencia la objetividad procedimental, aquella que ocurre cuando un proceso es dirimido de tal manera que siempre produce el mismo resultado o que no requiere juicio individual al momento de considerar su corrección, la objetividad concordante, aquella que remite a los acuerdos intersubjetivos, sin discusión previa, respecto de una descripción de una experimentación o de un juicio, y la objetividad interactiva, aquella que surge a partir de una discusión llevada adelante por grupos con formación pertinente y apropiadamente constituidos.

Como fuera, para los intereses de estas reflexiones resulta fundamental analizar los sentidos de objetividad relacionados con la posición de quienes investigan. Sobre este punto, Douglas observa que la objetividad distanciada o separada [detached] refiere a la prohibición de utilizar valores en lugar de evidencia, sean personales o grupales, y a la necesidad de reservar el rol indirecto en el análisis general de evidencia en favor de sostener o rechazar afirmaciones empíricas. Lamentablemente, la concepción de objetividad distanciada aparece estrechamente vinculada con la de libertad valorativa, razón que explica por ejemplo que en las publicaciones científicas no haya menciones a los procesos de toma de decisiones, como si eso volviera más objetiva a la investigación, cuando lo que efectivamente sucede es que se ocultan las alternativas y los valores que ayudaron a tomar cada decisión haciendo que la propia investigación se torne imposible de examinar por sus pares. Por el contrario, y sigo aquí una observación de Elliot $(2011,382)$, la indi- 
cación de explicitar esos valores constituye para Douglas un principio ético de la experticia científica. En favor de mantener esa diferencia crucial entre objetividad distanciada y libertad valorativa Douglas añade entonces un sentido de objetividad como neutralidad valorativa en tanto posición reflexivamente equidistante entre los valores que puedan entrar en conflicto al momento de decidir qué se debe hacer. Por supuesto, este sentido no es deseable en todos los casos pues adoptar un lugar inicialmente imparcial respecto de la incidencia de valores sexistas o racistas en la producción de conocimiento científico no es aceptable, toda vez que ese tipo de posiciones reflejan debates sociales saldados. En este marco, la investigación sobre el índice de abuelidad también respeta ambos criterios de objetividad: respecto de la objetividad distanciada, he indicado ya que el rol de los valores no-epistémicos es indirecto, esto es, no adoptan el rol de razones para la caracterización de datos o para la aceptación o rechazo de evidencia. Por tanto, su inclusión no implica ningún riesgo de subjetividad, entendiendo por esto algún tipo de influencia en detrimento de la objetividad o confiabilidad de la investigación (Douglas 2004b, 468). Respecto de la objetividad como neutralidad, entiendo que en un caso como el analizado la asunción de un lugar imparcial respecto de la incidencia de los valores morales, sociales y políticos al analizar las consecuencias no-epistémicas de la investigación es una asunción indeseable $-\mathrm{y}$ ha sido efectivamente evitada por quienes llevaron adelante la investigación, como puede leerse en las líneas finales del artículo:

[d]esde la perspectiva científica, nuestro objetivo es proporcionar el apoyo técnico que comprende una parte de este esfuerzo [de restitución de la identidad]. Desde la perspectiva de partícipes en los movimientos de derechos humanos, compartimos con nuestros colegas las preocupaciones en curso acerca del bienestar de los niños. (di Lonardo et al. 1984, 346).

\section{Conclusiones}

En este artículo he propuesto un análisis de la investigación que condujo a la elaboración del índice de probabilidad de inclusión de abuelidad utilizado al momento de determinar si una persona es efectivamente nieta de un conjunto determinado de abuelos/as y surgido a partir de la necesidad de obtener respaldo científico para el proceso de restitución de víctimas de apropiación ilegal durante la última dictadura en Argentina. Para ello, me he valido del marco teórico elaborado por Heather Douglas, particularmente respecto a los criterios por los que la incidencia de valores no-epistémicos en la investigación científica es legítima y deseable. Así, he recuperado su triple desafío al ideal en cuanto punto de partida sólido para señalar las limitaciones de la pretendida libertad valorativa de la ciencia. Asimismo, me he detenido en su argumento acerca de las consecuencias no epistémicas de las investigaciones como justificación de la necesidad de incorporar valores no-epistémicos y en su distinción entre roles directos e indirectos de los valores en ciencia, distinción que se propone como alternativa al ideal de ciencia libre de valores.

Revista de Humanidades de Valparaíso, 2020, No 16, 255-275

(c) $(1)(9)$ CC BY-NC-ND 
Una vez planteado el marco teórico, he avanzado en una reconstrucción de la investigación señalada a efectos de responder por qué han desestimado la obtención de una prueba con marcadores genéticos que arrojaba un índice de probabilidad de comprobación de la hipótesis de 96,2\% (índice que sería aceptado en la gran mayoría de las investigaciones científicas) y han avanzado hasta obtener una prueba con otros marcadores genéticos que arrojan un 99, 9\% de probabilidad de inclusión. En mi lectura, esa diferencia se explica por referencia a los valores sociales, políticos y morales con los que se consideran las consecuencias del riesgo inductivo de la investigación en el marco de la elaboración de políticas públicas por parte del Estado argentino en torno a la restitución de nietas/os apropiadas. Ahora bien, esos valores sociales, políticos y morales mantienen una incidencia indirecta pues influyen sólo en la consideración de la suficiencia de la evidencia y no en otros puntos de la investigación, como la caracterización de los datos -puntos para los que Douglas rechaza el rol directo de los valores sociales, morales y políticos. Además, esa incidencia es tal que la investigación antedicha se mantiene en los marcos de objetividad señalados por Douglas, especialmente porque no se emplean valores como evidencia o como pre-condiciones que deben ser cumplidas por la investigación y porque las posiciones asumidas por quienes llevaron adelante la investigación no supusieron un sentido irreflexivo de neutralidad sino que asumieron una posición de compromiso explícito con los derechos humanos, cumpliendo con el principio ético de la experticia científica. En virtud de lo dicho, la tarea de di Lonardo et al. constituye, en mi interpretación, un caso de necesaria y legítima integración entre valores epistémicos y no-epistémicos para un buen desarrollo de la investigación científica.

Para finalizar me interesa retomar una observación de John Dewey, con quien iniciara estas páginas. En su opinión, la comprensión y manipulación de la ciencia y de su poderosa capacidad de transformar el mundo es uno de los problemas más complejos de la civilización contemporánea, de modo que considerar el lugar y la función de la ciencia en el amplio y continuo curso de experiencia una tarea más propiamente filosófica que cualquier análisis auto-contenido de la ciencia en cuanto tal (Dewey 1985, 20). Esto implica comprender las relaciones entre los problemas morales, epistémicos e institucionales y revisar las relaciones entre ciencia y política. Frente a las posiciones herederas de la versión standard en filosofía de las ciencias y a pesar de las críticas señaladas, el enfoque de Douglas se muestra doblemente interesante pues, por un lado, ofrece herramientas teóricas para analizar la dimensión valorativa de la ciencia y abonar los enfoques que discuten desde diversas perspectivas el ideal de ciencia libre de valores $\mathrm{y}$, por el otro, permite analizar la incidencia de valores políticos, sociales y morales en investigaciones concretas, con todos los recaudos teóricos para evitar la ciencia politizada pero asumiendo la necesidad de integrar ciencia y valores no-epistémicos. Todos estos asuntos, en definitiva, conducen a una labor siempre abierta: reflexionar sobre los fines de la ciencia, en clave eminentemente política. 
Genética humana y el valor de los valores no-epistémicos en la restitución de identidad en Argentina

Livio Mattarollo

\section{Referencias bibliográficas}

Abuelas de Plaza de Mayo (2020). Casos Resueltos. Obtenido de: https://www.abuelas.org.ar/ caso/buscar?tipo=3. Consulta: 20/06/2020.

Anderson, E. (1995). Knowledge, Human Interests, and Objectivity in Feminist Epistemology. Philosophical Topics, 23(2), 27-58. https://doi.org/10.5840/philtopics199523213

Anderson, E. (2004). Uses of Value Judgments in Science: A General Argument, with Lessons from a Case Study of Feminist Research on Divorce. Hypatia, 19(2), 2-24. https://doi.org/10.1111/j.1527-2001.2004.tb01266.x

Amantze Regueiro, S. (2010). Análisis genético para la identificación de niños apropiados: construcción política y científica de la "naturaleza" y el parentesco. Revista Estudios Feministas, Florianópolis, 18(1), 11-32. https://doi.org/10.1590/S0104-026X2010000100002

Brown, M. (2013). Values in Science. Beyond Underdetermination and Inductive Risk. Philosophy of Science, 80(5), 829-839. https://doi.org/10.1086/673720

CONADEP (1984). Informe "Nunca Más". Buenos Aires: Eudeba.

Daston, L. (1992). Objectivity and the Escape from Perspective. Social Studies of Science, 22, 597-618. https://doi.org/10.1177/030631292022004002

di Lonardo A. M., Darlu P., Baur M., Orrego C., King M. C. (1984). Human Genetics and Human Rights: Identifying the Families of Kidnapped Children. American Journal of Forensic Medicine and Pathology, 5(4), 339-347. https://doi.org/10.1097/00000433-198412000-00011

Dewey, J. (1985). Context and Thought. En Jo Ann Boydston (ed.), The Collected Works of John Dewey, 1882-1953. The Later Works, 1925-1953. Volume 6: 1931-1932. Essays, Reviews, and Miscellany, pp. 3-21. Carbondale: Southern Illinois University Press.

Douglas, H. (2000). Inductive Risk and Values in Science. Philosophy of Science, 67(4), 559-579. https://doi.org/10.1086/392855

Douglas, H. (2004a). Border Skirmishes Between Science and Policy: Autonomy, Responsibility, and Values. En P. Machamer \& G. Wolters (eds.), Science, Values and Objectivity, pp. 220244. Pittsburgh: Pittsburgh University Press.

Douglas, H. (2004b). The Irreducible Complexity of Objectivity. Synthese 138(3), 453-473. https://doi.org/10.1023/B:SYNT.0000016451.18182.91

Douglas, H. (2007). Rejecting the Idea of Value-Free Science. En H. Kincaid, J. Dupré, \& A. Wylie (eds.), Value-free science? Ideals and Illusions, pp. 120-139. Oxford \& New York: Oxford University Press.

Douglas, H. (2009). Science, Policy and the Free-Value Ideal. Pittsburgh: University of Pittsburgh Press. https://doi.org/10.2307/j.ctt6wrc78

Douglas, H. (2014). The Moral Terrain of Science. Erkenntnis, 79(S5), 961-979. https://doi. org/10.1007/s10670-013-9538-0 
Genética humana y el valor de los valores no-epistémicos en la restitución de identidad en Argentina Livio Mattarollo

Douglas, H. (2016). Values in Science. En P. Humphreys (ed.). The Oxford Handbook in the Philosophy of Science, pp. 609-630. Oxford \& New York: Oxford University Press. https://doi. org/10.1093/oxfordhb/9780199368815.013.28

Echeverría, J. (2002). Ciencia y valores. Barcelona: Ediciones Destino.

Elliott, K. (2011). Direct and Indirect Roles for Values in Science. Philosophy of Science, 78(2), 303-324. https://doi.org/10.1086/659222

Elliott, K. (2013). Douglas on Values: From Indirect Roles to Multiple Goals. Studies in History and Philosophy of Science, 44(3), 375-83. https://doi.org/10.1016/j.shpsa.2013.06.003

Gómez, R. J. (2014). La dimensión valorativa de las ciencias. Hacia una filosofía politica. Bernal: Universidad Nacional de Quilmes.

Hempel, C. G. (1965). Science and Human Values. En Aspects of Scientific Explanation and other Essays in the Philosophy of Science, pp. 81-96. New York: The Free Press.

Hicks, D. J. (2014). A New Direction for Science and Values. Synthese, 191(14), 3271-3295. https://doi.org/10.1007/s11229-014-0447-9

Kitcher, P. (2001). Science, Truth, and Democracy. Oxford \& New York: Oxford University Press. https://doi.org/10.1093/0195145836.001.0001

Kitcher, P. (2011). Science in a Democratic Society. Oxford: Oxford University Press. https://doi. org/10.1163/9789401207355_003

Kourany, J. A. (2010). Philosophy of science after feminism. Oxford: Oxford University Press. https://doi.org/10.1093/acprof:oso/9780199732623.001.0001

Lacey, H. (1999). Is Science Value Free? Values and Scientific Understanding. London \& New York: Routledge. https://doi.org/10.4324/9780203983195

Longino, H. E. (1990). Science as Social Knowledge. Princeton, N.J: Princeton University Press.

Longino H. E. (1996) Cognitive and Non-Cognitive Values in Science: Rethinking the Dichotomy. En L. H. Nelson, J. Nelson (Eds). Feminism, Science, and the Philosophy of Science, pp. 39-58. Dordrecht: Springer. https://doi.org/10.1007/978-94-009-1742-2_3

Longino, H. E. (2002). The Fate of Knowledge. Princeton, N. J.: Princeton University Press.

Mattarollo, L. (2020). Valoración e investigación en el continuo de la experiencia. Desde el pragmatismo de John Dewey al debate sobre el ideal de ciencia libre de valores. Tesis de posgrado (Doctor en Filosofía). Universidad Nacional de La Plata. Facultad de Humanidades y Ciencias de la Educación. https://doi.org/10.35537/10915/91055

Naciones Unidas (1993). Declaración sobre la protección de todas las personas contra las desapariciones forzadas. URL: https://undocs.org/es/A/RES/47/133 Consulta: 25/06/2020.

Olivé, L. (2000). El bien, el mal y la razón. México DF: Paidós.

Penchaszadeh, V. B. (1992). Abduction of Children of Political Dissidents in Argentina and the Role of Human Genetics in Their Restitution. Journal of Public Health Policy, 13(3), 291305. https://doi.org/10.2307/3342729 
Genética humana y el valor de los valores no-epistémicos en la restitución de identidad en Argentina Livio Mattarollo

Penchaszadeh, V. B. (2015). Ethical, legal and social issues in restoring genetic identity after forced disappearance and suppression of identity in Argentina. Journal of Community Genetics, 6(3), 207-213. https://doi.org/10.1007/s12687-015-0219-3

Reisch, G. A. (2009). Cómo la Guerra Fría transformó la filosofía de la ciencia: Hacia las heladas laderas de la lógica. Bernal: Universidad Nacional de Quilmes.

Rudner, R. (1953). The scientist qua scientist makes value judgments. Philosophy of Science, 20(1), 1-6. https://doi.org/10.1086/287231

Rooney, P. (1992). On Values in Science: Is the Epistemic/Non-Epistemic Distinction Useful? PSA: Proceedings of the Biennial Meeting of the Philosophy of Science Association, 1, 1322. https://doi.org/10.1086/psaprocbienmeetp.1992.1.192740

Steel, D., Whyte, K. P. (2012). Environmental Justice, Values, and Scientific Expertise. Kennedy Institute of Ethics Journal, 22(2), 163-182. https://doi.org/10.1353/ken.2012.0010

Wullf, G. (2008). Las Abuelas y la Genética. El aporte de la ciencia en la búsqueda de los chicos desaparecidos. Buenos Aires: Editorial Abuelas de Plaza de Mayo. 
